Published: M. Rauterberg (ed.) Proceedings of ICEC $20043^{\text {rd }}$ International Conference on Entertainment Computing, Eindhoven, The Netherlands, 1-3 September, 2004. Springer-Verlag, pp.377-385.

\title{
Social Translucence of the Xbox Live Voice Channel
}

\author{
Martin R. Gibbs, Kevin Hew, Greg Wadley \\ Department of Information Systems \\ The University of Melbourne \\ Parkville, VIC 3010, Australia \\ martinrg@unimelb.edu.au, kevinhew@hotpop.com, \\ gwadley@unimelb.edu. au
}

\begin{abstract}
In this paper we use the concept of 'social translucence' to understand users' initial reaction to, and use of, the voice communication channel provided by Xbox Live. We found that although users expected voice to be an advance over text-based communication, in practice they found voice difficult to use. In particular, users experienced difficulties controlling the voice channel and these difficulties are indicative of usability and sociability problems with the configuration of the voice channel in some Xbox Live games. We argue that game developers will need to address these problems in order to realize the potential of voice in online multiplayer videogames. We believe these problems can be addressed by designing the voice channel so that socially salient information is made available to participants according to interactional affordances and constraints that are sensibly designed and well understood by users.
\end{abstract}

\section{Introduction}

Launched in the US in 2002 and in Europe and Australia in 2003, Microsoft's Xbox Live is a networking system allowing multiplayer gaming via the Internet using Xbox videogame consoles. Xbox Live brings together a novel cluster of features that includes: multiplayer gaming over the Internet; a console rather than a PC platform; voice communication using microphone and earphone headset; and a 'gamertag' system for the central management of online identity. In particular, and of interest in this paper, Xbox Live combines voice communication with a virtual environment, connecting users via broadband Internet for the purpose of computer mediated game play.

Typically, online communication has relied on typed text messages, and text is still the dominant medium for communication in computer games. However, Xbox consoles do not include keyboards, but users of Xbox Live can communicate with other players by voice in real time. This new mode of interaction makes Xbox Live an interesting technology to study. In this paper we address the following research question: How do users react to their first encounter with voice communication in Xbox Live? To collect data we used a constructive interaction technique for usability testing [11], and focus group discussions, to gauge users' initial reactions to voice in multiplayer, online console videogames. We found that while participants expected voice to be an advance over text-based communication, in practice many found voice difficult to use 
Published: M. Rauterberg (ed.) Proceedings of ICEC $20043^{\text {rd }}$ International Conference on Entertainment Computing, Eindhoven, The Netherlands, 1-3 September, 2004. Springer-Verlag, pp.377-385.

due to inadequate facilities provided in the games tested to control what is heard and what is sent over the voice channel. Drawing on the concepts of sociability and 'social translucence' in computer mediated communication, we argue that this lack of control hindered the ability of participants in the study to ascertain socially significant information. As a result, their capacity to engage in convivial computer-mediated social interaction was inhibited. We conclude with a discussion of our findings and their implications for the design and configuration of voice communication in online multiplayer video games.

\section{Sociability and Social Translucence}

In the context of designing technologies to support online communities, sociability has been described as 'planning and developing social policies and supporting social interactions' [12: p605]. Sociability and usability are closely related yet usefully separated concepts for analyzing and designing technologies that support online communities. While usability concerns the interaction between a user and a technological artifact, sociability concerns the interactions between people that occur via the artifacts. Sociability includes the policies and social norms as well as the design features of the mediating technology that govern and influence behaviour online. While sociability concerns the interactions between people, the mediating technology must be usable if it is to support convivial social interactions [6].

We believe sociability is an important construct for understanding all computermediated communication, including instances of computer supported cooperative play [14] such as those found in online multiplayer game play. As Bannon has argued [1], consideration of the social layer of computing systems is crucial in systems design. This is further supported by Kutti [8], who argues that human-computer interaction needs to be understood in terms of social factors as well as the task-human-artifact dynamic. To successfully enable cooperative, sociable interaction in digital environments, close attention should be paid to the forms of social interaction afforded by mediating technologies. In terms of designing online multiplayer computer games, sociability emphasizes the importance not only of planning for the interaction between the player and the game, but also planning for the interactions between the people playing the game [14].

Xbox Live has a number of features designed to manage the sociability of the system. These include a fixed user-selected pseudonym ('gamertag') for player identity and accountability, player rankings for many games, and a friends list to allow players to find other players with whom they prefer to play. In addition, Xbox Live has introduced a voice channel for communication between players, and Microsoft has mandated that all Xbox Live games implement this form of communication. The configuration of the voice channel - the manner in which it is implemented within a particular game - and its resulting usability as a convivial medium, will significantly impact the sociability of games within the Xbox Live network.

We believe that voice communication can be a valuable addition to the game playing experience and the sociability of the game environment if it is 'socially translu- 
Published: M. Rauterberg (ed.) Proceedings of ICEC $20043^{\text {rd }}$ International Conference on Entertainment Computing, Eindhoven, The Netherlands, 1-3 September, 2004. Springer-Verlag, pp.377-385.

cent'. Social translucence is a design approach that emphasizes the importance of making socially significant information visible to participants in digital environments [5].

Social translucence has two dimensions. First, socially translucent systems make socially salient information available to participants. This visibility enables participants to be aware of others and their actions. This awareness helps bring the social rules, norms and customs that govern participants' actions and interactions into effect. It also allows participants to be held accountable for their actions. Visibility, awareness, and accountability are crucial to the sociability of digital environments because they enable participants to structure their interactions with one another in coherent and sensible ways [5]. Thus, in the design of sociable online games, it is important for users to be able to easily discern and identify the other users with whom they are interacting, and to be able to readily associate activity and actions with particular game identities and personas.

Second, socially translucent systems are not transparent; communication within these systems is constrained. In the physical world, 'physics' constrains communication and determines who can receive what information or communication in what circumstances. People use these constraints as a resource in social interaction. For example, it is far easier to see and hear a person standing by one's side than it is to see and hear someone across a crowded room and this proximity constraint is routinely used in a host of communicative acts such as raising and lowering one's voice depending on one's intended audience and social situation. Similarly, in designing socially translucent systems, attention needs to be paid to the 'physics', or rules, that govern communication within these digital environments. Furthermore, if participants share an awareness of the constraints that underlie the visibility of socially significant information in a digital environment they can use this understanding as a resource for structuring their social interactions. Thus, we would argue, sociability within online game environments is enhanced by making socially salient information available to participants according to interactional affordances and constraints that are sensibly designed and well understood by those involved.

\section{Research Approach}

Our research was conducted with six groups of three volunteers. Each group participated in one, two-hour research session. At the start of each session, participants completed a background questionnaire. They were then asked to play multiplayer games with other players over the Internet using Xbox Live. We observed their interaction both with other players via the network, and with other participants in the room. After the participants played games for an hour, a researcher led them in a focus group discussion. Open-ended questions sought the participants' opinions about their first encounter with Xbox Live. Questions focused on topics such as the voice headset, and playing online. The sessions were recorded using a single video camera directed at the participants. This data was analyzed to identify common themes across the groups 
Published: M. Rauterberg (ed.) Proceedings of ICEC $20043^{\text {rd }}$ International Conference on Entertainment Computing, Eindhoven, The Netherlands, 1-3 September, 2004. Springer-Verlag, pp.377-385.

through a process of open-coding, then closed-coding to refine and confirm prevalent themes.

Participants were aged between 18 and 27 years and both genders were represented. Participants reported that, on average, they played PC-based computer games more often than console games (6 hours per week versus 2 hours). Two participants played for over 20 hours per week. Only three participants played console games more often than computer games. Participants liked several different types of game. The most popular genres were first-person shooters (13), action games (13), sports (10), strategy games (10) and role-playing games (9). All participants owned or regularly used a computer and had Internet access.

Two games were used: MotoGP and Unreal Championship. MotoGP is a motorbike racing game that is packaged with every Xbox Live starter kit. MotoGP incorporates a 'lobby' area, outside the main racing arena, where players waiting for their race to start can converse in a party-line fashion. During a race, a proximity-based algorithm is used, allowing players to talk to each other if they are close together on the track. Unreal Championship is a first-person shooter allowing either all-against-all or team-based modes of play. In all-against-all play, each player can hear all other players speak. In the team games, all players on a given team can speak to and hear each other.

Groups were used in the observation sessions to encourage participants to express their views and describe their experience. This approach was based on the constructive interaction technique developed by O'Malley et al. [11] for usability testing. Also known as co-discovery learning [7], this technique aims to create an environment in which participants verbalize their thoughts more readily than does an individual 'thinking aloud' to a researcher [10]. The focus group discussions that followed each game playing session provided participants with an opportunity to discuss and reflect upon their initial encounter with Xbox Live. These discussions were used to provide insights into participants' opinions, feelings and attitudes about their experience playing with Xbox Live [9].

\section{Findings}

The primary purpose of connecting consoles via the Internet is to increase the opportunity to play with other people, rather than computer-generated opponents. The participants in our study all valued the social interactions entailed in playing with other people and cited three major reasons why they preferred to this form of play: unpredictability; fairness; and enjoyment of competition. Human players were regarded as being unpredictable because they are creative and do not follow a pre-programmed 'script'. Some participants felt that the computer 'cheated' or had an unfair advantage over them, particularly at high difficulty settings, because computer opponents could be unfairly fast, accurate, or omniscient. Participants reported deriving more satisfaction from beating human opponents than computer-generated ones, 'even though you can't see the person.' Knowledge that there was another person in the game, even though they were not co-present, substantially increased enjoyment of the game. 
Published: M. Rauterberg (ed.) Proceedings of ICEC $20043^{\text {rd }}$ International Conference on Entertainment Computing, Eindhoven, The Netherlands, 1-3 September, 2004. Springer-Verlag, pp.377-385.

Clearly users want to play against other people. One could assume that any feature that enhanced the social interactions of online multiplayer gaming would increase users' enjoyment. We might expect the addition of voice communication to game play to enhance social interaction. However, the voice system, as it was configured in the games used, often seemed to detract from the enjoyment of the game as a social experience. In the following sections we report on users' reaction to voice in Xbox Live.

Usefulness of Voice Communication - Perception vs. Practice. Participants expected that voice communication would enrich their multiplayer gaming experience. They expected voice would be an improvement on text-based messaging. In particular, many of our participants thought that voice would be beneficial in tactical team-based games, by better supporting the coordination of team members:

I quite like the headset, the fact that you can talk. Especially in Unreal where, well the fact that you're supposed to work as a team and you can sort of do that quite well. I think it's better than having to type or anything like that.

An important advantage participants in the study cited for using the voice headset was freeing hands from the task of typing messages. Participants felt this would allow them to maintain control over their character's movements while they communicated with other players:

[Voice] was good because with network games on computers you have to type messages and that interferes with the game. But, [voice] was good because it didn't interfere with what you were doing.

This assumed advantage created by voice over other forms of in-game communication was mentioned by the majority of participants. We, as researchers, certainly expected voice to positively augment the gaming experience. However, many participants stopped using the voice headset during observation sessions, effectively rejecting the technology. In practice, it would seem, our participants found voice communication to be unhelpful and not particularly useful or useable in the games used in this study due to a number of difficulties they experienced with the use of voice. These difficulties can be understood as being problems of channel control: controlling what is received, and what is sent, over the voice channel. We discuss these problems next.

Controlling What is Heard Through the Voice Channel. Participants reported two problems with what they heard through the headset: 'noise' over the voice channel, and an inability to identify who was talking.

Participants felt that a lot of what was sent over the voice channel resembled noise more than conversation. Four types of noise were present on Xbox Live. First, the voice channel included speech that was not intended for the listener. Participants could hear conversations that they were not part of. Due to the configuration of the voice channel in the games used in this study, these conversations were as prominent in the voice channel as utterances directed at the participants. Second, participants overheard conversations that were apparently between groups of users co-located around consoles distant on the network. By leaving the microphone active, parts of these conversations were broadcast over the network. Third, participants encountered non-conversational sound such as television, vocalized motorbike sounds, and background music that was apparently transmitted unintentionally. Fourth, sometimes the 
Published: M. Rauterberg (ed.) Proceedings of ICEC $20043^{\text {rd }}$ International Conference on Entertainment Computing, Eindhoven, The Netherlands, 1-3 September, 2004. Springer-Verlag, pp.377-385.

non-conversational sound encountered by participants appeared to have been sent intentionally, to limit the usability of the channel for others. Some of our participants referred to this as 'spam'. Examples included loud incoherent speech, relentless trashtalking, and music at a volume that suggested the sender's headset microphone was placed close to a hi-fi speaker. In response to noise of this kind, one participant suggested a preference towards typing over voice. He reasoned, 'that bit of effort in typing text just means you don't get crap on the airwaves.' That is, the ease with which noise could be generated over the voice channel was seen as a negative feature once users had experienced a variety of irritating and anti-social examples of its usage.

Participants also had problems identifying who was talking on the voice channel. They were unable to link the voice they heard via the headset to the name of the user or the user's avatar on the screen:

It's hard to identify who you are talking to. I mean you can't get the sense of community if you are connected to all these people, [but] you can't really see them or you don't know who you are talking to.

This was particularly pronounced in Unreal Championship, where the voice channel was configured in a broadcast, party-line mode, so that everyone could hear each other equally, regardless of their position in the game world. This effectively 'disembodied' the voice, because the volume and clarity of transmission was unrelated to proximity. MotoGP uses a coarse proximity-based algorithm that switches voices on or off according to the proximity of players on the racetrack. As a result, voices suddenly appeared, mid-sentence, with no sense of a person approaching or receding. Participants found these voices just as disembodied as those in Unreal Championship.

The ability of online gamers to connect socially with one another will depend on their ability to identify who is talking. However it is also important for a user to know who is listening, and we discuss this issue next.

Controlling What is Sent Over the Voice Channel. Participants experienced two problems involving transmissions they made over the voice channel: controlling who could hear what they said, and knowing whether their utterances were heard.

When participants spoke into the headset microphone, they could neither control nor know who was listening to their transmission. Participants desired the ability to direct their messages to specific people, but could not find this functionality. One said, 'I didn't know who I was talking to. There wasn't functionality to select who I was talking to.' And another, in a different session, stated: 'One thing I didn't like was not knowing who you could or couldn't speak to.'

Participants experienced the voice channel as chaotic and out of control. One participant suggested: 'It might be good if you could direct the speech, so you can specify before you talk who you want to hear it and who you don't want to hear it.' Suggestions of this kind were made frequently by participants.

A related problem was participants' uncertainty over whether their utterances were being heard by the intended recipients. Participants often did not receive a response from other players in the game, and were uncertain whether they were being heard. One participant was heard to say: 'I'm hanging around the flag. Where are you? Can you hear me? Hello?' During other sessions participants repeatedly asked: 'Is anyone there?' or 'Hello, hello?' in attempts to prompt a response from other players. Par- 
Published: M. Rauterberg (ed.) Proceedings of ICEC $20043^{\text {rd }}$ International Conference on Entertainment Computing, Eindhoven, The Netherlands, 1-3 September, 2004. Springer-Verlag, pp.377-385.

ticipants received no visual cues or other indications as to whether their transmissions were being sent or heard successfully.

\section{Discussion}

Our findings suggest that the potential to engage in computer mediated social interaction was hindered by the problems with the voice channel participants faced while using Xbox Live. Many participants rejected the voice headset, due to their inability to control both what they heard over the headset and who they were speaking to via the headset. Because voice is the only channel on Xbox Live through which players can communicate with other players, the rejection of the voice headset removed the participants' ability to engage other players in conversation. When participants did attempt to use the headset, they had problems identifying who was talking, and who they were talking to. Participants could not link utterances to people or their avatars and thus had difficulty interacting with other players on the Xbox Live network. This absence of social cues created disorder and chaos in the voice channel.

The inability to ascertain socially salient information - such as the identity of the person who was speaking, and an awareness of who was listening - reflects a lack of social translucence on Xbox Live. Kellogg and Erickson [5] suggest that we approach the design of socially translucent systems by first asking what properties of the physical world support graceful, coherent and nuanced communication between people. For example, if a metaphor of face-to-face conversation were used to guide the implementation of voice within a game, the volume of utterances would attenuate over distance, visual cues would be available to help determine who was speaking, and listeners would be able to perceive the direction utterances were coming from. Other features of a game environment might also constrain the ability to communicate, and limit the availability of social cues, such as only being able to communicate with other players within the same room or within close proximity. Other metaphors may also be appropriate in guiding how voice is implemented, depending on the game and its genre.

Xbox Live is new and our choice of games to use in this study was limited. It may be that the games used were not the most apt to encourage voice communication. The fast-paced and competitive nature of the games used may have limited social interaction. Social interactions are more prominent in multiplayer RPGs, and voice may be more suitable to this genre of game. However, it has been suggested that voice communication will detract from immersion in RPGs [2]. The genre of games in which voice will be useful and heavily used remains an open question at this early stage in the life of Xbox Live.

Our results suggest two directions for future research. Further work is required to determine how to implement socially translucent voice communication in online multiplayer video games. We are aware that researchers at Microsoft are working to improve the implementation of voice in Xbox Live games [13]. In pursuing this line of enquiry it will be necessary to bear in mind that different genres of games, and different styles of game play, will have different requirements in this regard.

Second, while our study has been useful for gauging participants' initial reaction to Xbox Live and for evaluating usability problems with current implementations of voice 
Published: M. Rauterberg (ed.) Proceedings of ICEC $20043^{\text {rd }}$ International Conference on Entertainment Computing, Eindhoven, The Netherlands, 1-3 September, 2004. Springer-Verlag, pp.377-385.

communication in games, further investigation of how voice communication is taken up and used by the game-playing community is also needed. Contextual interviews [3] with players, and ethnographically informed observations [4] of game play in settings where it naturally occurs, such as lounge-rooms, LAN cafes, and online digital game environments, should offer valuable insights into the adoption and appropriation (or otherwise) of the voice channel in online multiplayer games. We intend to pursue these avenues of inquiry in the future.

\section{Conclusion}

The research reported in this paper represents the first stage of a longer study into the adoption, adaptation and integration of voice communication media into everyday online multiplayer gaming practice. It has provided an early look into how users react to their first encounters with Xbox Live. Our key findings were: (a) the participants' ability and willingness to engage socially with others on Xbox Live was hindered by the poor usability of the voice channel; (b) the voice channel's poor usability in the games we tested was due to lack of control over what is sent, and what is received, over the channel; (c) while participants expected voice communication to be an advance over text-based communication, in practice they often rejected it.

Our finding suggest that the current implementation of voice in some Xbox Live games is socially 'opaque' in that it is difficult to infer socially relevant information that would aid interaction, such as who is saying what to whom. In order for online videogames to be convivial places for social interaction we need to design systems that make socially salient information available to participants through carefully designed interactional affordances and constraints. The availability of socially relevant information should help participants to be aware of what is occurring in the social milieu of the online games they are playing, and accountable for their actions as a consequence of the public knowledge of that awareness. Furthermore, the rules or 'physics' governing the ways in which this socially significant information is made available need to be both sensible and easily discerned by participants. If this is the case, these rules can be used as a resource in social interaction by participants. We believe that voice communication in online games can be a valuable addition to both game play and the sociability of these digital environments. However, to realize this potential, the voice channel in online games needs to be implemented as a socially translucent mode of communication.

\section{Acknowledgements}

We would like to thank Conner Graham for his helpful advice, Steve Goschnick for supporting our use of the IDEA Lab, and the Interaction Design Group at The University of Melbourne for constructive criticism of earlier versions of this work. 
Published: M. Rauterberg (ed.) Proceedings of ICEC $20043^{\text {rd }}$ International Conference on Entertainment Computing, Eindhoven, The Netherlands, 1-3 September, 2004. Springer-Verlag, pp.377-385.

\section{References}

1. Bannon, L.: From Human Factors to Human Actors: The Role of Psychology and HumanComputer Interaction Studies in System Design. In: Greenbaum, J., Kyng, M. (eds): Design at Work. Lawrence Erlbaum, Hillsdale, NJ (1991) 25-44

2. Bartle, R. Not Yet You Fools! Game+Girl=Advance. 28 July (2003): http://www.gamegirladvance.com/archives/2003/07/28/not_yet_you_fools.html

3. Beyer, H., Holtzblatt, K.: Contextual Design: Defining Customer-Centered Systems. Morgan Kaufmann, San Francisco (1998)

4. Blomberg, J., Burrell, M., et al.: An Ethnographic Approach to Design. In: Jacko, J., Sears, A. (eds): The Human-Computer Interaction Handbook. Lawrence Erlbaum, Mahwah, NJ (2003) 964-986

5. Erickson, T., Kellogg, W.: Social Translucence - An Approach to Designing Systems that Support Social Processes. ACM Trans. Computer-Human Interaction, 7 (2000) 59-83

6. Hew, K., Gibbs, M., Wadley, G.: Usability and Sociability of the Xbox Live Voice Channel. In Pisan, Y.: Proceedings Australian Workshop on Interactive Entertainment (IE2004), Creative and Cognitive Studios Press, Sydney (2004) 51-58

7. Kennedy, S. Using Video in the BNR Usability Lab. ACM Special Interest Group on Computer-Human Interaction Bulletin, 21, 2, (1989) 92-95

8. Kutti, K.: Activity Theory as a Potential Framework for Human-Computer Interaction Research. In: Nardi, B. (ed.): Context and Consciousness. MIT Press, London (1996) 17-44

9. Krueger R.: Focus Groups: A Practical Guide for Applied Research. Sage, Thousand Oaks CA (1994)

10. Nielsen, J.: Usability Engineering. Academic Press, Boston (1993)

11. O’Malley, C., Draper, S., et al.: Constructive Interaction: A Method for Studying HumanComputer Interaction. In: Shackel, B. (ed.): Proceedings of Interact 84, North Holland, Amsterdam (1984) 269-274

12. Preece, J., Maloney-Krichmar, D.: Online Communities: Focusing on Sociability and Usability. In: Jacko, J.A., Sears, A. (eds): The Human-Computer Interaction Handbook. Lawrence Erlbaum Mahwah, NJ (2003) 596-620

13. Terrano, M.: Lessons from Life: Designing More Immersive Games. Paper presented at the Australian Game Developers Conference, Melbourne, 20-23 November, (2003)

14. Wadley, G., Gibbs, M., Hew, K., Graham, C.: Computer Supported Cooperative Play, "Third Places" and Online Videogames. In Viller, S., Wyeth, P. (eds): Proceedings 2003 Australasian Computer Human Interaction Conference (OzCHI 2003), Ergonomics Society of Australia, Canberra (2003) 238-241 